\title{
THE NONREVERSIBILITY OF ADSORPTION-DESORPTION OF DIELDRIN AND LINDANE ON CHITIN IN SEAWATER
}

\author{
J. M. SANTANA-CASIANO, J. PEREZ-PEÑA and M. GONZALEZ-DAVILA \\ Universidad de Las Palmas de Gran Canaria. Facultad de Ciencias del Mar. \\ Departamento de Química. P.O. Box 550. Las Palmas. España.
}

(Received 13 March 1991; in final form, 31 July 1991)

\begin{abstract}
Adsorption and desorption of dieldrin and lindane on chitin were investigated in seawater batch tests as a function of chitin concentration, temperature, $\mathrm{pH}$ and salinity. For chitin concentrations ranging from $0.5 \mathrm{~g} \mathrm{l}^{-1}$ to $12.5 \mathrm{~g} \mathrm{l}^{-1}$, the pesticide concentrations were varied from $4 \mu \mathrm{g}^{-1}$ to $65 \mu \mathrm{g} \mathrm{l}^{-1}$ for dieldrin and from $40 \mu \mathrm{g}^{-1}$ to $680 \mu \mathrm{g}^{-1}$ for lindane. Both dieldrin and lindane show adsorption-desorption hysteresis at low chitin concentration. At high chitin concentrations $\left(m>6.25 \mathrm{~g}^{-1}\right.$ for dieldrin and $m>10 \mathrm{~g}^{-1}$ for lindane) both pesticides exhibit reversible behaviour. However, only lindane adsorption is affected by chitin concentration. These types of behaviour remain fixed in prewashed chitin. However, an increase in the temperature and a decrease in the salinity made the process become reversible. A resistant-reversible two component model has been applied to account for these types of behaviour and provides a way to explain most of the observed effects by defining mass independent distribution coefficients.
\end{abstract}

KEY WORDS: Adsorption, desorption, dieldrin, lindane, chitin, seawater.

\section{INTRODUCTION}

In aquatic environments, one of the fundamental processes controlling the behaviour, fate and distribution of persistent organic compounds is the exchange of these chemicals between dissolved and sorbed states. Clay and suspended organic particulate matter are considered important for adsorption, but very little is known about the actual adsorption-desorption process ${ }^{1-3}$. The scavenging of chlorinated hydrocarbons by organic particulates could provide a means of transporting these persistent pollutants through the water column and for concentrating them in sediments and in benthic and planktonic detritus-feeding organisms. The strong particle-binding tendency of persistent organic chemicals such as DDT, lindane, and PCBs is well known, and its importance has been stressed many times ${ }^{4,5}$.

Chitin (poly-N-acetyl-D-glucosamine), as well as its deacetylated derivative chitosan, is a constituent of the natural dissolved and particulate organics and part of fungi and bacteria. It is consequently abundant in the natural environment. The extent of chlorinated hydrocarbon sorption on chitin is, thus, a critical component in the evaluation of its fate in water systems. However, very little is known about the interaction of pesticides with this polymer ${ }^{6.7}$. 
In this work we present the results of the adsorption-desorption of dieldrin and lindane on chitin in seawater. The studies have been done taking into account two important facts of these processes: first, the reversibility or nonreversibility of the adsorption reaction ${ }^{2,8}$ between the lindane and dieldrin and the natural chitin, and second, the effect of chitin concentration on the adsorption-desorption process ${ }^{3,9,10}$. Results of studies at different $\mathrm{pH}$, temperature, and salinity values were also analysed.

\section{EXPERIMENTAL}

The chitin powder used was derived from crab shells and obtained from Sigma (Sigma, Chemical Co., MO, U.S.A.). The surface area $\left(0.5 \pm 0.03 \mathrm{~m}^{2} \mathrm{~g}^{-1}\right)$ for the bulk chitin and the amphoteric properties of the surface of chitin $\left(\mathrm{pK}_{a 1}=4.4 \pm 0.2\right.$ and $\mathrm{pK}_{a 2}=6.4 \pm 0.3$ ) were determined in a previous work ${ }^{11}$. Preliminary adsorption studies with various size fractions from less than $88 \mu \mathrm{m}$ to $250 \mu \mathrm{m}$ give similar values to those of the bulk, which was used in all the following studies. Stock solutions of dieldrin ( $98 \%$ ) and lindane ( $99 \%$ ) (Supelco Inc, Bellefonte, PA, U.S.A.) were prepared in pesticide grade hexane $7.5 \mathrm{ppm}$ and $75 \mathrm{ppm}$, respectively.

All batch sorption studies (in triplicate) were conducted by preequilibrating the dieldrin (ranging from $4 \mu \mathrm{g} \mathrm{l}^{-1}$ to $65 \mu \mathrm{g} \mathrm{l}^{-1}$ ) (solubility ${ }^{12} 2 \cdot 10^{2} \mu \mathrm{g}^{-1}$ ) and the lindane (ranging from $40 \mu \mathrm{g}^{-1}$ to $685 \mu \mathrm{g} \mathrm{l}^{-1}$ ) (solubility ${ }^{12} 7.8 \cdot 10^{3} \mu \mathrm{g}^{-1}$ ) with $40 \mathrm{ml}$ of $0.45 \mu \mathrm{m}$ filtered and hexane-preextracted seawater $(\mathrm{S}=36.52 \%$ ) from Gran Canaria (Canary Islands). After $24 \mathrm{~h}$ preequilibration period, the chitin was added (from $0.5 \mathrm{~g} \mathrm{l}^{-1}$ to $12.5 \mathrm{~g}^{-1}$ ) and the glass stoppered centrifuge glass tubes (volume: $50 \mathrm{~cm}^{3}$ ) were shaken in a controlled temperature bath for $24 \mathrm{~h}$ adsorption and $24 \mathrm{~h}$ desorption time periods when equilibrium conditions were desired. After shaking, the supernatant was centrifuged for $15 \mathrm{~min}$ at $6,000 \mathrm{rpm}$, after which $25 \mathrm{ml}$ of supernatant was withdrawn and extracted with $10 \mathrm{ml}$ of hexane. In the recovery studies, repeated extractions showed the first extraction removed over $97 \%$ of both pesticide masses. In all the dieldrin studies, the extract was reduced to $1 \mathrm{ml}$ in rotavapor at $40^{\circ} \mathrm{C}$ and at $360 \mathrm{~mm} \mathrm{Hg}$ controlled vacuum pressure. Extracted samples were analysed and quantified by gas chromatography with a linearized ${ }^{63} \mathrm{Ni}$ ECD detector. A SPB-5 (Supleco Inc) $30 \mathrm{~m}$-fused silica capillary column was used with $\mathrm{N}_{2}$ carrier gas at a linear velocity of $25 \mathrm{~cm} \mathrm{~s}^{-1}$. Samples were analysed isothermally $\left(200^{\circ} \mathrm{C}\right)$ using on-column injection and a detector temperature of $300^{\circ} \mathrm{C}$.

The pesticide mass was derived from triplicate measurements by comparing their peak area responses to a standard curve prepared with standard solution of the pesticide in the same experimental conditions. The coefficients of variation of these triplicate measurements were about $1 \%$. It was assumed that the difference between the pesticide mass added and the pesticide mass in liquid phase at the end was the result of chitin adsorption.

For the desorption, the following procedure was repeated several times: the supernatant was replaced with contaminant-free seawater, shaken again for 24 hour periods, when equilibrium conditions were desired, recentrifuged, and the new sunernatant analvsed. 


\section{RESULTS AND DISCUSSION}

\section{Adsorption-desorption kinetics}

The adsorption-desorption rate of dieldrin and lindane on chitin $\left(2 \mathrm{~g} \mathrm{l}^{-1}\right.$ and $6.25 \mathrm{~g} \mathrm{l}^{-1}$, respectively) was determined at initial concentrations of $18.75 \mu \mathrm{g} \mathrm{l}^{-1}$ (dieldrin) and $187.5 \mu \mathrm{g} \mathrm{l}^{-1}$ (lindane) in seawater. For the adsorption studies, pesticide solutions were equilibrated with the chitin for periods ranging from $5 \mathrm{~min}$ to $92 \mathrm{~h}$ and for the desorption studies, after $24 \mathrm{~h}$ equilibration periods, the contaminated chitin was preequilibrated with contaminant-free seawater for periods ranging from 5 to $74 \mathrm{hr}$. A comparison of the lindane and the dieldrin adsorbed and desorbed from solution by chitin is shown in Figure 1. The data show that initial uptake is rapid mainly for lindane and a $3 \mathrm{~h}$ period for the adsorption of lindane and $10 \mathrm{~h}$ periods for the adsorption of dieldrin were enough to reach equilibrium. For the desorption experiments, the process is essentially complete in a few minutes and much longer desorption times do not produce notable effects. $24 \mathrm{~h}$ equilibrium periods both for adsorption and desorption experiments were used in all the studies. The present results do suggest kinetically rapid adsorption-desorption reactions, similar to other studies on organic partitioning between the sediment and water phases ${ }^{2,13}$.

\section{Isotherms for dieldrin and lindane}

Figure 2 shows the results of single adsorption-desorption of dieldrin conducted at two chitin concentration and this is typical of all the results obtained over the study.

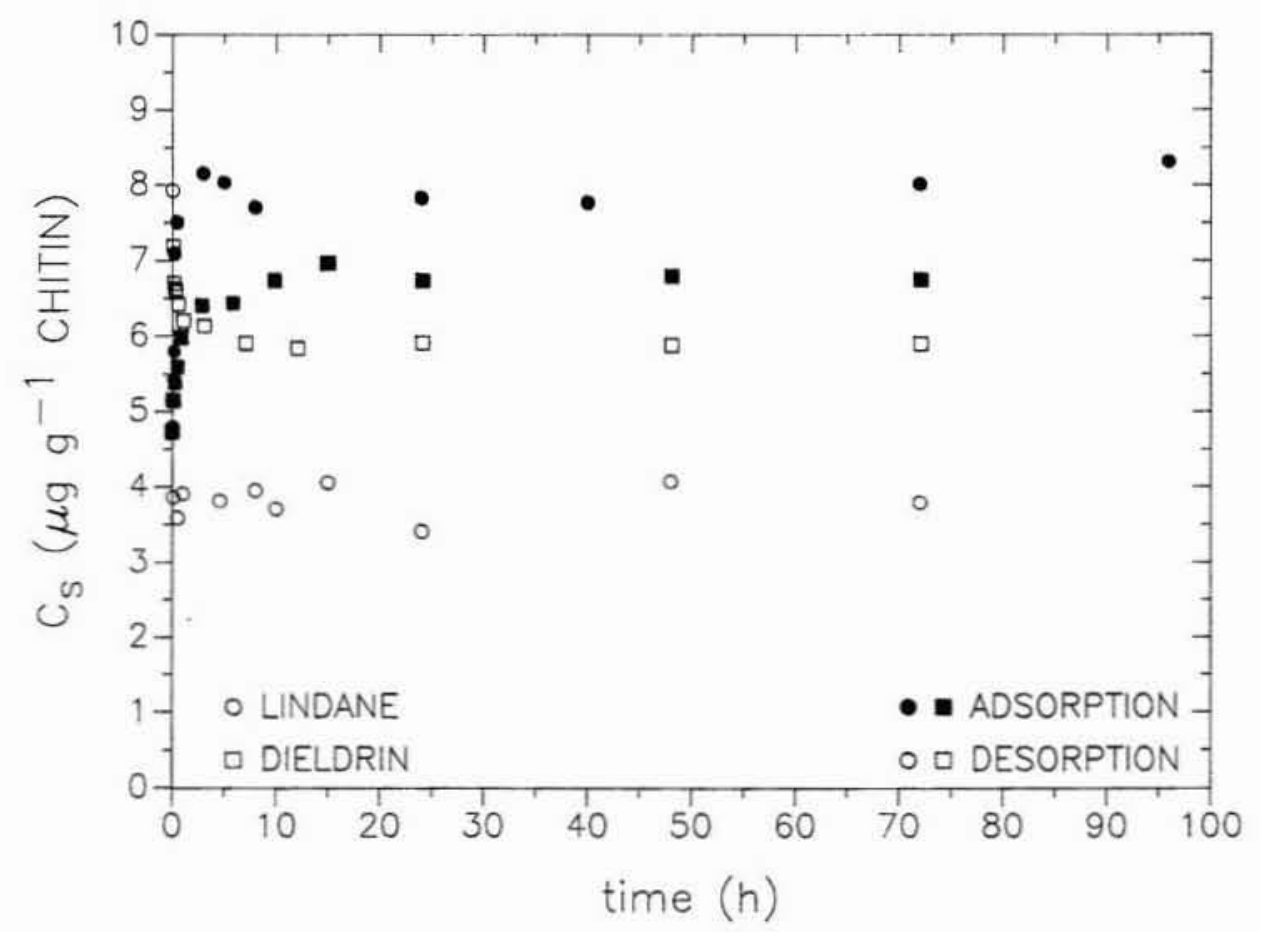

Figure 1 Adsorption-desorption kinetics of dieldrin and lindane on chitin. For the desorption kinetics, adsorption equilibrium time was fixed at $24 \mathrm{~h}$. 


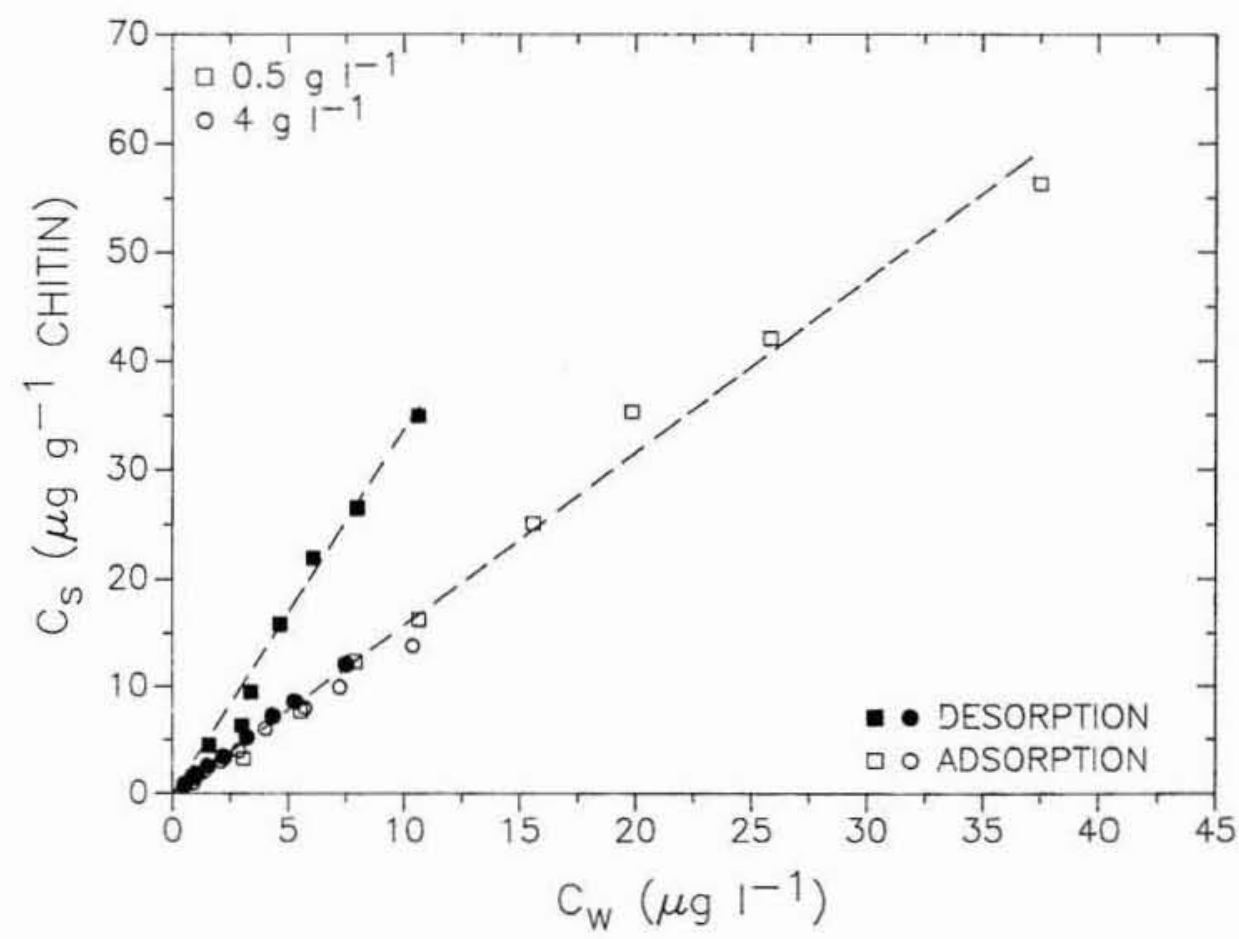

Figure 2 Dieldrin adsorption-desorption isotherms on chitin $\left(m=0.5 \mathrm{~g} \mathrm{l}^{-1}\right.$ and $\left.m=4 \mathrm{~g} \mathrm{l}^{-1}\right)$. Data are averages of three replicates.

The behaviour shown by the lindane adsorption-desorption is also depicted in Figure 3. Around $60 \%$ of dieldrin is adsorbed per gram of chitin and it is 25 -times more strongly adsorbed on chitin than lindane. Only $25 \%$ on average of the lindane dissolved in seawater is adsorbed per gram of chitin. The increased uptake of dieldrin over lindane may be due to the asymmetry of the dieldrin molecule which may influence its electron density causing increased polarization compared to lindane. The increased polarity may result in stronger binding forces between the dieldrin and the chitin particles.

Reasonable linearity over the range of concentrations studied can be observed for both pesticides, and the near zero intercept of a linear regression of the data supports the validity of a linear partitioning model.

$$
C_{s a}=K_{a} C_{w a}
$$

where $C_{s a}$ and $C_{w a}$ are the adsorbed and aqueous equilibrium concentration and $K_{a}$ is the partition coefficient for the adsorption.

Similarly, the desorption points fall on a straight line, and a desorption isotherm which corresponds with the previous adsorption reaction, can be defined as

$$
C_{s d}=K_{d} C_{w d}
$$

with partition coefficient, $K_{d}$, and $C_{s d}$ and $C_{w d}$ the adsorbed and aqueous equilibrium concentration for the desorption studies. The results illustrated in Figures 2 and 3 
Table 2 Lindane adsorption-desorption partition coefficients and the resistant-reversible component partition coefficients to chitin in seawater $\left(22^{\circ} \mathrm{C}\right.$ and $36.52 \%$ salinity)

\begin{tabular}{lllll}
\hline [Chitin] $\left(\mathrm{gl}^{-1}\right)$ & $K_{a}\left(\mathrm{lg}^{-1}\right)$ & $K_{d}\left(\mathrm{lg}^{-1}\right)$ & $K_{o}\left(\mathrm{lg}^{-1}\right)$ & $K_{x}\left(\mathrm{lg}^{-1}\right)$ \\
\hline 2.5 & $0.080 \pm 0.004$ & $0.430 \pm 0.002$ & $0.039 \pm 0.002$ & $0.043 \pm 0.002$ \\
4 & $0.067 \pm 0.004$ & $0.234 \pm 0.028$ & $0.028 \pm 0.002$ & $0.041 \pm 0.003$ \\
6.25 & $0.062 \pm 0.003$ & $0.106 \pm 0.004$ & $0.018 \pm 0.002$ & $0.047 \pm 0.001$ \\
$6.25^{* 1}$ & $0.079 \pm 0.007$ & $0.186 \pm 0.003$ & $0.032 \pm 0.008$ & $0.046 \pm 0.001$ \\
$6.25^{* 2}$ & $0.042 \pm 0.002$ & $0.043 \pm 0.007$ & $0.000 \pm 0.001$ & $0.044 \pm 0.001$ \\
$6.25^{\# 1}$ & $0.053 \pm 0.003$ & $0.075 \pm 0.009$ & $0.006 \pm 0.002$ & $0.049 \pm 0.001$ \\
$6.25^{\# 2}$ & $0.049 \pm 0.002$ & $0.052 \pm 0.004$ & $0.001 \pm 0.001$ & $0.050 \pm 0.001$ \\
7.5 & $0.060 \pm 0.002$ & $0.089 \pm 0.006$ & $0.012 \pm 0.001$ & $0.048 \pm 0.002$ \\
10 & $0.055 \pm 0.002$ & $0.074 \pm 0.004$ & $0.009 \pm 0.001$ & $0.045 \pm 0.002$ \\
12.5 & $0.048 \pm 0.002$ & $0.060 \pm 0.002$ & $0.009 \pm 0.001$ & $0.041 \pm 0.004$ \\
\hline
\end{tabular}

* Values corresponding to lindane adsorption-desorption at $5^{\circ} \mathrm{C}(1)$ and $45^{\circ} \mathrm{C}(2)$.

"Values corresponding to lindane adsorption-desorption at salinity values of $25.56 \%$ (1) and $15.34 \%$ (2).

coefficients. This behaviour is affected by the chitin concentration and it is greater at lower chitin concentrations. The second effect is only observed for lindane. The lindane adsorption is affected by the adsorbent mass.

When after an initial desorption step we perform subsequent multiple desorptions the consecutive desorption isotherms are generated. Figure 4 shows the consecutive desorption isotherms for four dieldrin concentrations $\left(m=1 \mathrm{gl}^{-1}\right.$ chitin) showing significant nonreversibility as it has been reported for other systems ${ }^{2,8,14}$.

Gschwend and $\mathrm{Wu}^{15}$ and Baker et al ${ }^{16}$ have suggested that these complex sorptive phenomena are the result of artifacts associated with noncentrifugable or nonsettling

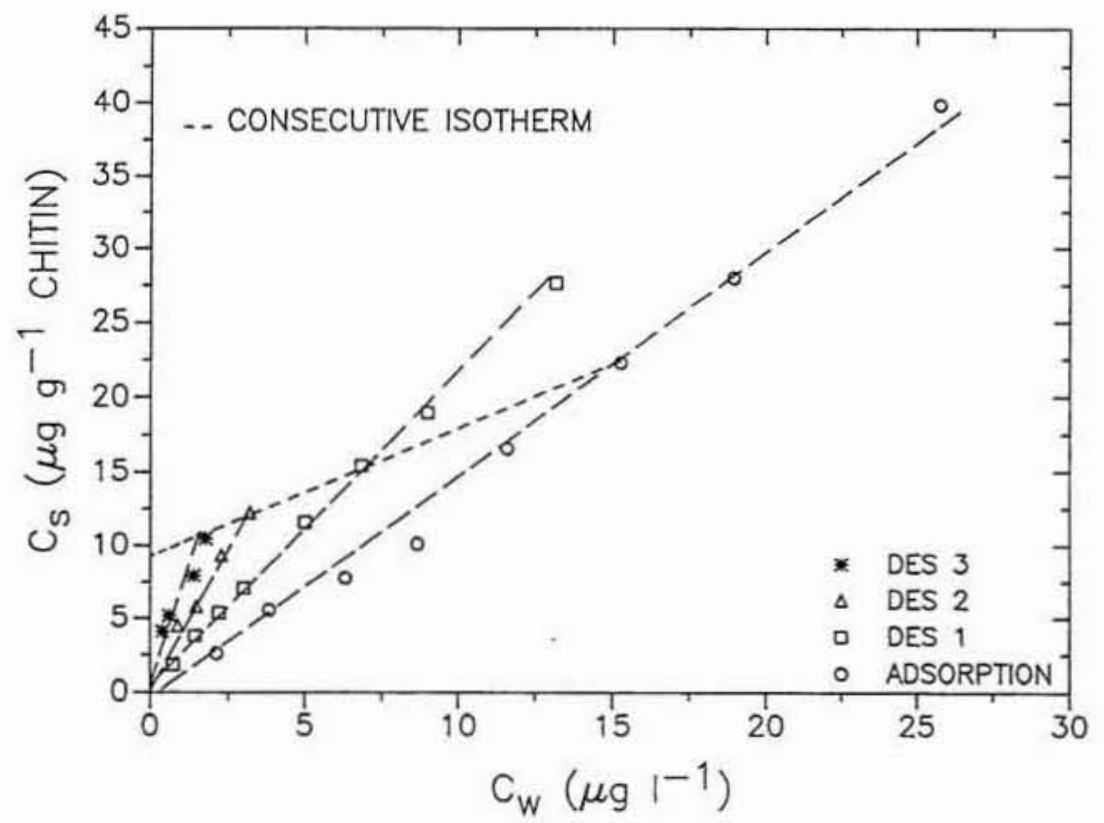

Figure 4 Single adsorption-desorption isotherms and consecutive isotherm of dieldrin on $4 \mathrm{~g}^{-1}$ chitin. From this Figure, the resistant-reversible componets of adsorption and desorption with the assumption of a linear consecutive desorption isotherm have been computed. 
microparticles and organic macromolecules released from the solids. When experiments were carried out using water-washed chitin following Gschwend and $\mathrm{Wu}^{15}$ (approximately five washes), these do not show significant effects on the nonreversibility of the adsorption-desorption process. As in other research works ${ }^{17.18}$ we can conclude that these effects are not exclusively responsible for the nonsingular isotherms shown by lindane and dieldrin adsorbed to chitin. However, when other factors, such as temperature and salinity, were changed, the adsorption-desorption hysteresis observed was dramatically reduced as we show below.

Adsorption of dieldrin and lindane is expected to take place via various mechanism due to the very heterogeneous surface of chitin that accounts for the nonreversibility observed. The chitin surface sites may be divided, as a first approximation, in easily accessible surface sites and less accessible internal voids, which can attach pesticide with differing strength. Next, we attempt to describe the adsorption-desorption process using a reversible-resistant two-component model ${ }^{2}$ which interprets incompletely reversible linear behaviour. Sorbed chemical, either at adsorption and at desorption equilibrium concentration is assumed to be the sum of a reversible sorbed component which readily adsorbs and desorbs reversibly and a resistant component which resists desorption over the time periods characteristic of experimental desorption equilibrium. The resistant component concentraction $C_{o}$ is defined as the extrapolated intersection of the consecutive isotherm and the ordinate, whilst the reversible component concentrations $C_{x a}$ and $C_{x d}$ corresponding to aqueous concentration $C_{w a}$ and $C_{w d}$, respectively are defined as the difference between the adsorbed pesticide concentration at the adsorption and desorption points and the resistant concentration

$$
\begin{aligned}
& C_{x a}=C_{s a}-C_{0} \\
& C_{x d}=C_{s d}-C_{0}
\end{aligned}
$$

The model assumes the reversible component concentrations $C_{x}$ are described by a unique linear isotherm $C_{x}=K_{x} C_{w}$ both for the adsorption and desorption reversible components whilst the resistant component concentration follows a linear isotherm $C_{o}=K_{o} C_{w a}$. These isotherms define the reversible component partition coefficient $K_{x}$ and the resistant component partition coefficient $K_{o}$.

Figure 5 shows for lindane adsorbed to $4 \mathrm{~g} \mathrm{l}^{-1}$ chitin concentration the determined linear isotherm for the resistant and reversible component concentrations $C_{o}, C_{x a}$ and $C_{x d}$, confirming the component model assumptions. Tables 1 and 2 show the results obtained for dieldrin and lindane, respectively, at all the chitin concentrations studied. These component partition coefficients $K_{x}$ and $K_{o}$ can also be calculated from the adsorption partition coefficient, $K_{a}$, and single desorption partition coefficient, $K_{d}$, by using the relationship ${ }^{2}$

$$
\begin{aligned}
& K_{o}=\frac{m K_{a}\left(K_{d}-K_{a}\right)}{1+m\left(K_{d}-K_{a}\right)} \\
& K_{x}=\frac{K_{a}}{1+m\left(K_{d}-K_{a}\right)}
\end{aligned}
$$




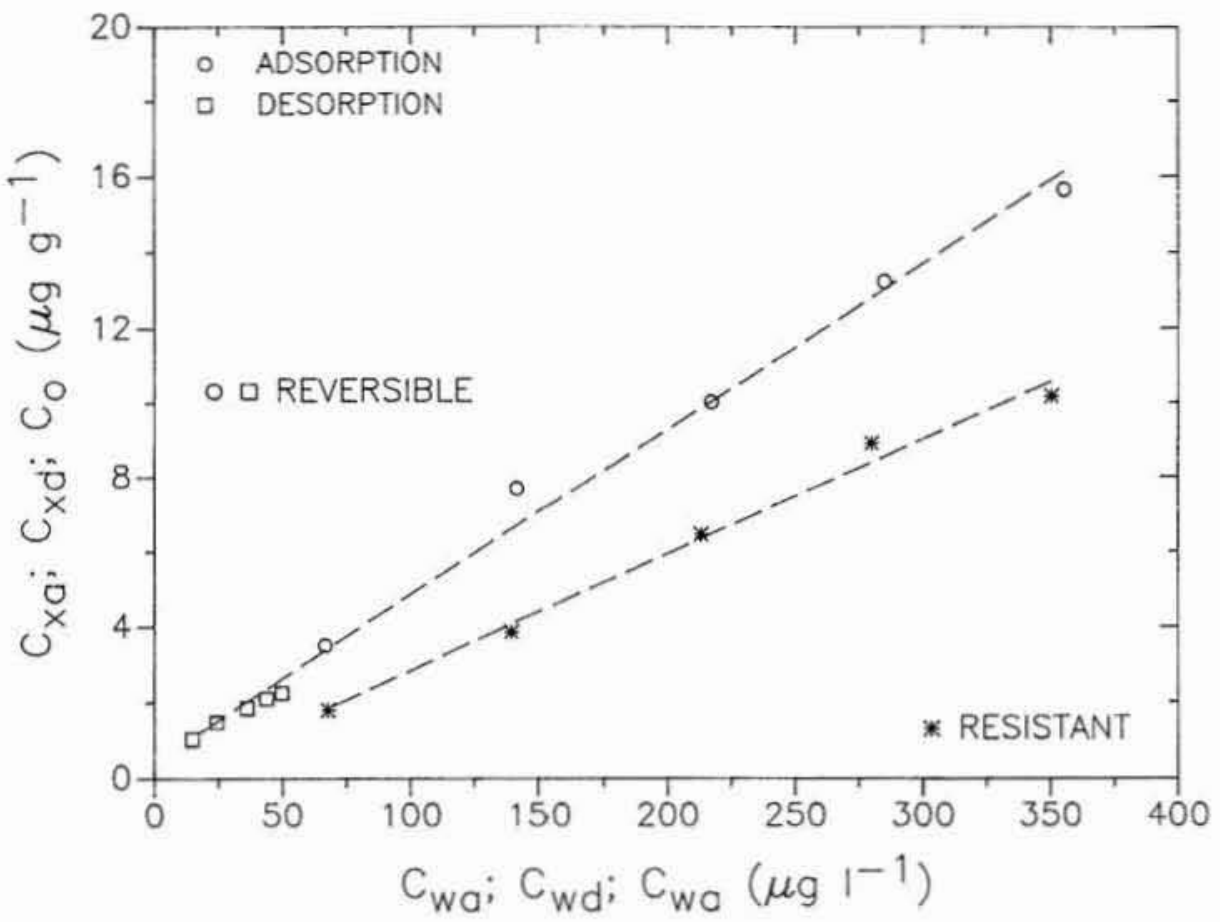

Figure 5 Resistant and reversible components and linear isotherms for lindane $\left(\mathrm{m}=4 \mathrm{~g} \mathrm{I}^{-1}\right)$.

These theoretical values are completely in agreement with those obtained by extrapolating the experimental results which confirm the model used.

The effect of the chitin concentration on the partition coefficients has been explained by applying this model both to lindane and dieldrin adsorption-desorption processes.

When the lindane reversible and resistant component partition coefficients are plotted versus mass (Figure 6), it can be clearly observed that while the reversible partition coefficient is not dependent on the chitin concentration, $K_{x}=0.045 \pm 0.004$, the resistant component partition coefficient is inversely related to chitin concentration, $K_{o}=v_{o} m^{-1}$, where $v_{o}=0.0978 \pm 0.007$ is defined as a dimensionless distribution coefficient for the resistant component. Using these two known values, the dependence of the adsorption and desorption partition coefficients for lindane as a function of the adsorbent concentration yields

$$
\begin{aligned}
& K_{a}=K_{x}+v_{0} m^{-1} \\
& K_{d}=K_{x}+v_{0} m^{-1}\left[1+\left(m K_{x}\right)^{-1}\right]
\end{aligned}
$$

If we define the index of irreversibility as the difference of $K_{d}$ and $K_{a}$,

$$
K_{d}-K_{a}=\frac{v_{0}}{m^{2} K_{x}}
$$




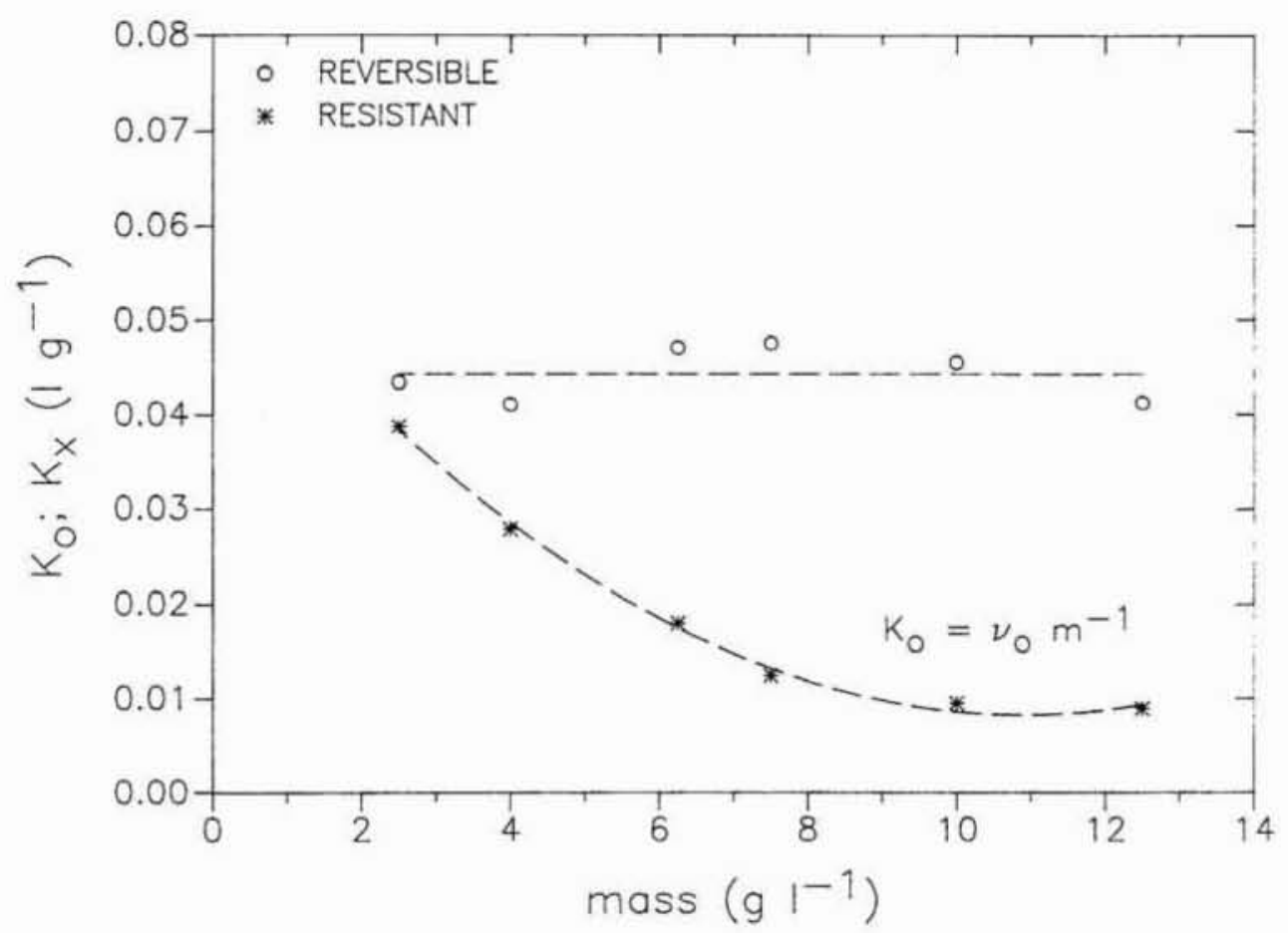

Figure 6 Lindane resistant, $K_{o}$, and reversible, $K_{x}$, component partition coefficient variation with adsorbent concentration. $K_{x}$ is quite constant in the concentration range studied.

where

$$
\begin{array}{ll}
K_{d}-K_{a} \rightarrow 0 & \text { as } m \rightarrow \infty \\
K_{d}-K_{a} \rightarrow \infty & \text { as } m \rightarrow 0
\end{array}
$$

These results explain the observed decrease of nonreversibility as the adsorbed mass concentration increase. It also gives the quantitative explanation for the almost reversible behaviour of the chitin at high concentrations.

When this same treatment is applied to dieldrin it is observed from Figure 7 that both reversible and resistant component partition coefficients are inversely related with the concentration

$$
\begin{aligned}
& K_{o}=v m^{-1} \\
& K_{x}=K_{x}^{\infty}-v m^{-1}
\end{aligned}
$$

where $v=0.3895 \pm 0.048$ is a dimensionless distribution coefficient for the resistant component and $K_{x}^{\infty}=1.437 \pm 0.0291 \mathrm{~g}^{-1}$, the partition coefficient describing the reversible adsorption-desorption of dieldrin to chitin.

Using these two parameters in the same way as before, we get

$$
\begin{aligned}
& K_{a}=K_{o}+K_{x}=K_{x}^{\infty} \\
& K_{d}=K_{x}^{\infty}+\frac{v}{m\left(m K^{\infty}-v\right)}
\end{aligned}
$$




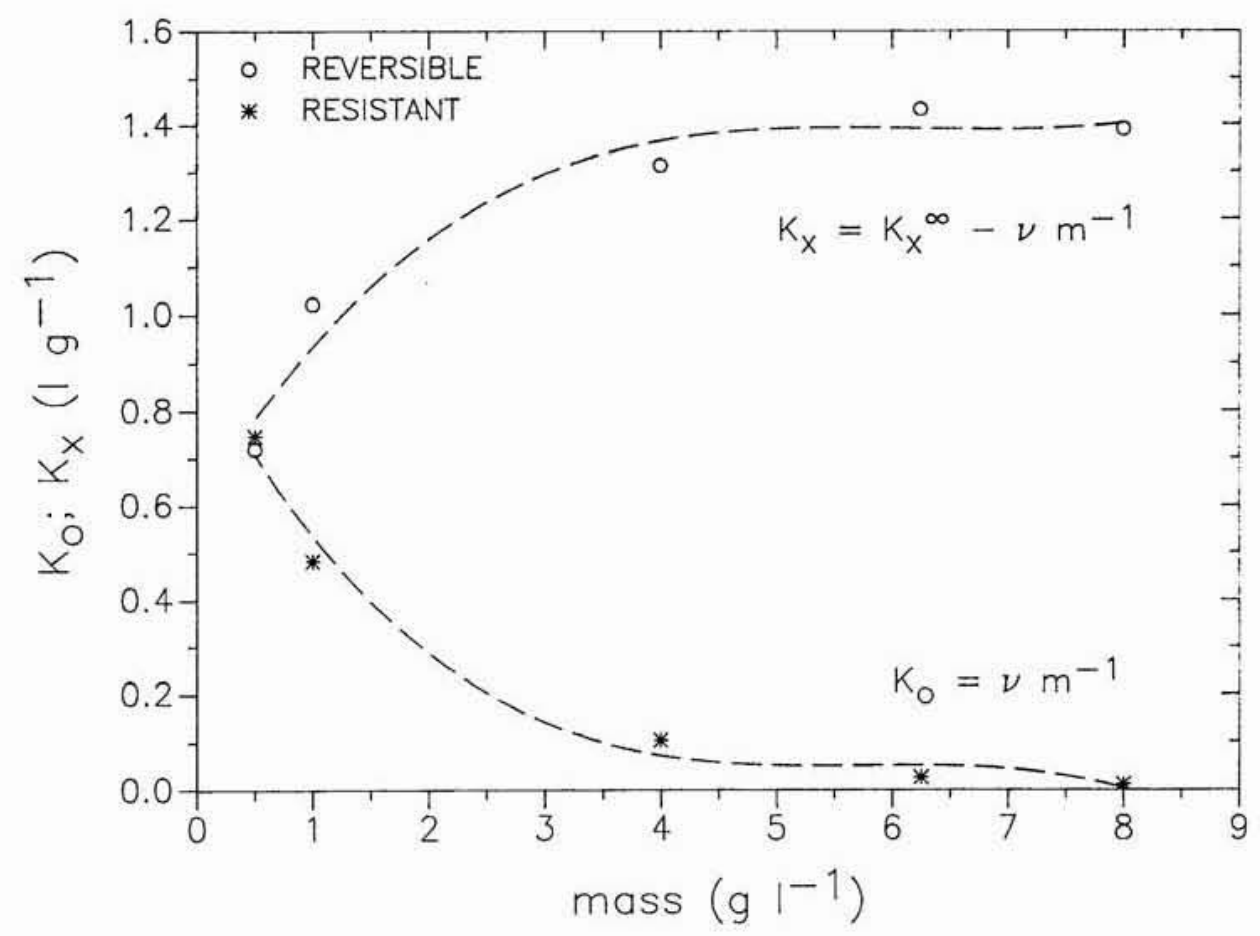

Figure 7 Dieldrin resistant and reversible component partition coefficient variation with chitin concentration. Both coefficients are inversely related with $m(\mathrm{~g} / \mathrm{l})$.

It demonstrates, first, that the adsorption partition coefficient is independent of the mass of chitin as can be seen in Figure 2, and second, that for the desorption partition coefficient $K_{d}$, the second term of the sum accounts for the nonreversibility observed. At high chitin concentrations $\left(m>6.25 \mathrm{gl}^{-1}\right)$, both, the adsorption and desorption partition coefficients are the same as, and equal to $K_{x}^{\infty}$.

\section{Effects of temperature, salinity and $\mathrm{pH}$}

According to the predicted value for an exothermic adsorption process, a decrease in the solution temperature, increases the adsorption process, both for dieldrin and lindane (Tables 1 and 2). Then again, an increase in temperature makes the process become reversible $\left(K_{d}=K_{a}\right)$. This demonstrates that the nonreversibility observed for the adsorption-desorption of these pesticides on chitin in seawater is not due to experimental artifacts. If this is true, at high temperatures, we can expect more nonsettling microparticles and dissolved macromolecules that account for the nonreversibility shown. Thus, we can conclude that the nonreversibility behaviour is a result of the adsorption-desorption reaction mechanism.

When the two-component model is applied to both systems (Tables 1 and 2) we observe that only the resistant component is affected. An increase in the temperature decreases the resistant component towards zero. At high temperatures, the attractive forces associated with the resistant places between the pesticide and chitin may decrease $^{9}$, resulting in greater desorption. 
When the studies were carried out at different salinities (Tables 1 and 2), the adsorption of dieldrin is not affected by changes in the ionic strength of the aqueous solution. However, it is observed that a decrease in salinity makes the process become reversible $\left(K_{a}=K_{d}\right.$ for $S=15 \%$ ). For lindane, both adsorption and desorption partition coefficients decrease as salinity decreases and at low salinities the process becomes reversible.

The application of the model to the experimental results shows for dieldrin (Table 1) that both, the resistant and the reversible component partition coefficients are affected by the ionic strength. The resistant component becomes zero while the reversible component partition coefficient reaches $K_{x}^{\infty}$ as salinity approaches $15 \%$. At lower salinity the adsorption-desorption reaction is not affected by changes in salinity. When the model is applied to the salinity effect on lindane adsorption-desorption (Table 2) it is observed that only the resistant component is affected, decreasing as salinity decreases.

Salinity may increase the adsorption as a result of the neutralization of the surface charge of chitin resulting from cation adsorption from seawater ${ }^{2}$. This interpretation of the salinity data would appear to be consistent with the $\mathrm{pH}$ results. The adsorption reaction, both for lindane and dieldrin, was affected by changes in the $\mathrm{pH}$ solution values. An increase in the adsorption partition coefficients at $\mathrm{pH}$ values lower than 4.5 was observed due to changes in the charge characteristics of the chitin surface which should have favored maximization of positive surface charge. It can account for higher attractive coulombic interaction which can be responsible for the nonreversibility observed at the salinity values of seawater. At high salinity values the solid aggregation and particle size may also be affected and may increase the partitioning on the resistant sites and, therefore increase the resistant component partition coefficient.

\section{CONCLUSION}

It has been demonstrated that both dieldrin and lindane are adsorbed on chitin in seawater. The increased uptake of dieldrin over lindane may be due to the asymmetry of the dieldrin molecule which may increase the polarity compared to lindane. The increased polarity may result in stronger binding forces between the dieldrin and the chitin particles. Both pesticides show a nonreversibility behaviour at low chitin concentration, and for lindane the adsorption is affected by the chitin concentration. Both effects are not consequences of the presence of nonsettling chitin microparticles and dissolved organic macromolecules. This effect dramatically is reduced both by increasing temperature or by decreasing salinity, which may affect the adsorption-desorption mechanism. These types of behaviour have been explained taking into consideration that the adsorption is the result of two components, one resistant and another reversible. These two components can relate the variations of the adsorption and desorption partition coefficients with the adsorbent concentration by defining mass independent distribution coefficients. Thus, the reversible-resistant model explains most of the observed effects on the adsorption-desorption processes. However, 
the mechanism that accounts for this behaviour is not clear yet, but the method described herein provides additional insight into the influence of aqueous-phase nodifications (e.g., alteration of the $\mathrm{pH}$, temperature and salinity).

\section{References}

1. R. H. Pierce, Jr., C. E. Olney and G. T. Felbeck Jr., Geochim. Cosmochim. Acta 38, 1061-1073 (1974).

2. D. M. Di Toro and L. M. Horzempa, Environ. Sci. Technol. 16, 594-602 (1982).

3. J. T. Coates and A. W. Elzerman, J. Contam. Hydrol. 1, 191-210 (1986).

4. R. Haque, In Dynamics, Exposure and Hazard Assessment of Toxic Chemicals, R. Haque Ed. (Ann Arbor Science: Ann Arbor, Ml, 1980).

5. L. L. Marking and R. A. Kimberle, Aquatic Toxicology Proc. Second Annual Symposium on Aquatic Toxicology (ASTMSTP 634; ASTM: Philadelphia, PA, 1977).

6. K. A. Lord, Biochem. J. 43, 72-79 (1948).

7. P. Davar and J. P. Wightman, In Adsorption from Aqueous solutions, (P. H. Tewari, Ed. Plenum, New York, 1981) pp. 163-177.

8. D. E. Peck, D. L. Corwin and W. J. Farmer, J. Environ. Qual. 16, 735-742 (1982).

9. D. M. Di Toro, L. M. Horzempa, M. M. Casey and W. Richardson, J. Great Lakes Res. 8, 336-349 (1982).

10. T. C. Voice, C. P. Rice and W. J. Weber Jr., Environ. Sci. Technol. 17, 513-518 (1983).

11. M. González-Dávila and F. J. Millero, Geochim. Cosmochim. Acta 54, 761-768 (1990).

12. A. S. Y. Chau and B. K. Afghan, In Analysis of Pesticides in Water (CRC Press, Boca Raton, FL, 1982), Vol. II, Chap. 1, p. 11.

13. W. C. Stenn, D. F. Paris and M. Baughman, Water Res. 12, 655-657 (1978).

14. P. A. Wahid and N. Sethunathan, J. Agric. Food Chem. 27, 1050-1053 (1979).

15. P. M. Gschwend and S.-C. Wu, Environ. Sci. Technol. 19, 90-96 (1985).

16 J. E. Baker, P. D. Capel and S. J. Eisenreich, Environ. Sci. Technol. 20, 1136-1143 (1986).

17. P. R. Jaffé, Environ. Sci. Health A21, 55-69 (1986).

18. P. J. Witkowski. P. R. Jaffé and R. A. Ferrara, J. Contam. Hvdrol. 2. 249-269 (1988). 